\title{
A Design Method of Metasurface using CCSR
}

\author{
A.A.M Ezanuddin ${ }^{1, *}$, and $A . H$ Ismail $^{1}$ \\ ${ }^{1}$ Fakulti Teknologi Kejuruteraan, Universiti Malaysia Perlis (UniMAP), Aras 1, Blok S2, Kampus \\ UniCITI Alam, 02100 Sungai Chucuh, Padang Besar Perlis, Malaysia.
}

\begin{abstract}
This research paper describes the steps used to design an alternative metasurface which is based on Circular Complementary Split Ring (CCSR) formation at $5.8 \mathrm{GHz}$. All the 3D modelling was conducted using specialized EM simulation software that utilizes Eigen algorithm to solve different frequency modes that can resonate within the CCSR structure itself. The final design will be then fabricated using a precise laser $\mathrm{CNC}$ machine to achieve a better dimension as closely as possible to the simulation dimension. By using laser $\mathrm{CNC}$, this step will able to overcome generic PCB design issue related to via's placement, signal integrity, power integrity and multilayer designs. Completed CCSR metasurface will be able to mitigate or block unwanted EM mode from flowing to its neighbouring sensitive area which can affect the entire component performance due to unseen harmonics.
\end{abstract}

\section{Introduction}

The systematic design of unit cells for a CCSR based surface, a particular class of metasurface, is presented here. The design of these unit cells is based on transmission-line theory and waveguide. The CCSR unit cells presented here are machined on a typical microwave laminate instead of many stacked, interspaced layers [1]. This simplifies fabrication and enables the scaling down of the metasurfaces to shorter wavelengths and are capable of synthesizing the full range of impedances required. In contrast to frequencyselective surfaces (FSSs) and traditional resonator arrays, which are on the order of a wavelength thick, these designs are only $\lambda / 10$ thick while incurring minimum reflections losses.

Figure 1 displays the basic Maxwell equations used to perform the required calculation There are four steps used in the simulation to produce a final result in the from Maxwell Grid equations. Whereas in Figure 2, it illustrates the basic material (Dielectric value of PCB) equations involved and it's corresponding mathematical variables used in calculating the transient solver for every $3 \mathrm{D}$ mesh grid of the proposed unit cell and full array unit cells. The transient purpose is to conduct integration calculations in iteration form until it can produce a stable and real signal waveform in scattering parameter format.

\footnotetext{
* Corresponding author: mezanuddin85@gmail.com
} 


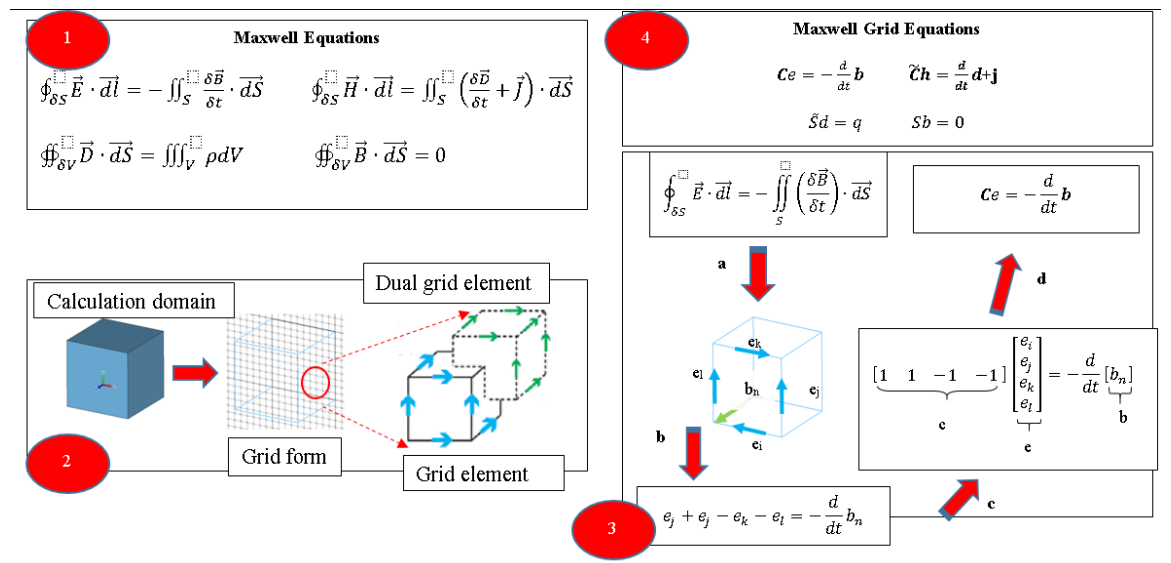

Fig. 1. Finite integration technique used within the 3D EM simulation technique and its steps

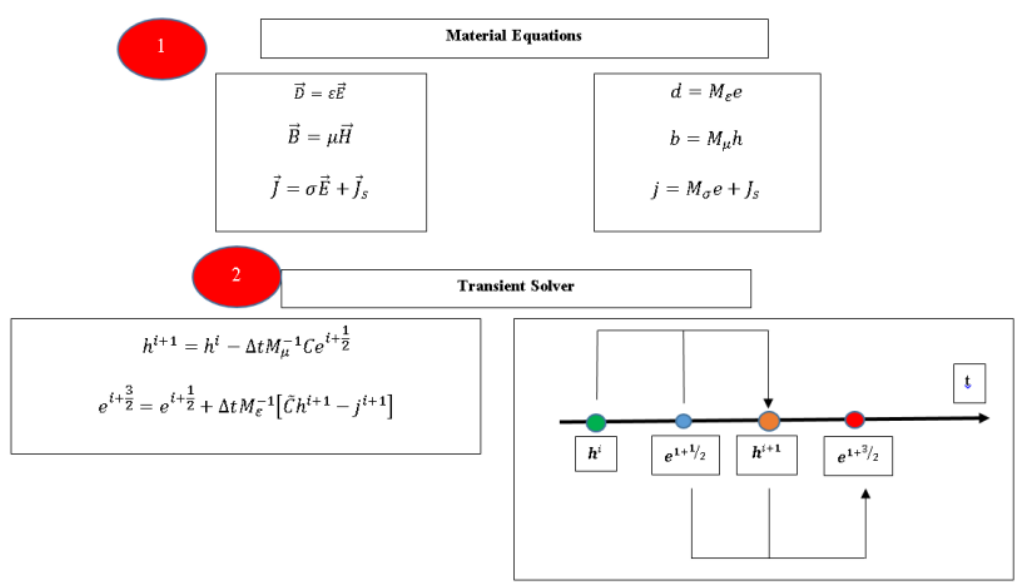

Fig. 2. 3D EM Transient solver for general S-Parameter simulation steps before moving to Eigen algorithm

\section{Initial simulation}

Provided that the unit cell is sub-wavelength, the CCSR unit cell can be approximated by an electric loop. In addition to this, the orientations of the CCSR unit cell must correspond with the polarizations of the input and output EM fields. This reflects the anisotropies of the impedances required to enforce the surface currents. Keeping this issue in mind, unit cells proposed here were synthesized using commercial full-wave 3D electromagnetics solver to obtain the required electric and magnetic impedances. Here infinite array analysis was used. This result makes sense in view of the transmission-line description of infinite array analysis [2].

Figure 3(a) and (b) show the proposed CCSR unit cell and one period of the simulated unit cell. Note that the $1 \mathrm{D}$ refraction is in the horizontal plane, and the unit cell is compatible with the field polarizations stipulated in these problems. The input and output electric field polarizations are vertically oriented. The input and output EM field polarizations are in the horizontal plane as can be seen in Figure 4. The structure was simulated using known waveguide model WR159 (operational between 4.90 to $7.05 \mathrm{GHz}$ ) This first unit cell was designed as an entirely printable metasurface, and are implemented 
on a FR4 substrate $\left[\varepsilon_{\mathrm{r}}=4.7, \tan (\delta)=0.0025\right]$. Final result shows that this CCSR can resonate well at $5.8 \mathrm{GHz}$.

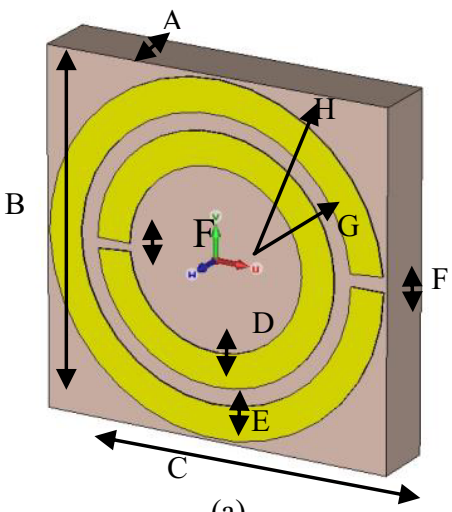

(a)

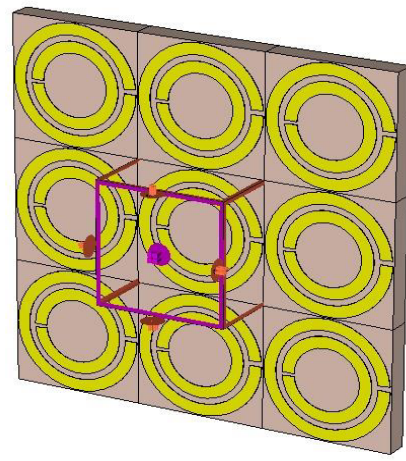

(b)

Fig. 3. CCSR unit cell a) the unit cell configuration are $A=1.6 \mathrm{~mm}, \mathrm{~B}=\mathrm{C}=9 \mathrm{~mm}, \mathrm{D}=\mathrm{E}=0.85$ $\mathrm{mm}, \mathrm{F}=3550$ (Angle), $\mathrm{G}=2.71 \mathrm{~mm}$ (radius) and $\mathrm{H}=4 \mathrm{~mm}$ (radius) and $\mathrm{b}$ ) unit cell with its boundary condition, two port setup was used here

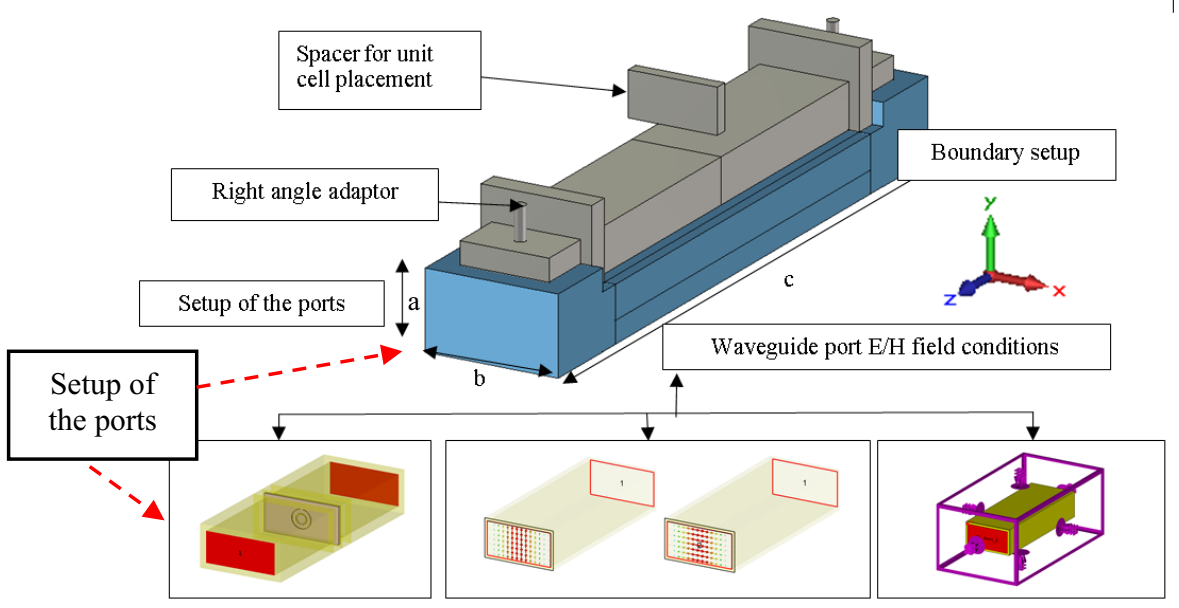

Fig. 4. Simulation of the CCSR unit cell inside a WR 159 waveguide to characterise its S-parameters from 5.85 to $8.2 \mathrm{GHz}$

\section{Fabrication and near-field measurement}

Now that all of the issues with the layout design have been resolved, the next step is to actually transfer to out the circuit layout and fabricate it. Layout editor was used to create a Gerber file, Gcode and Gerbtools ${ }^{\mathrm{TM}}$ to reorganize every gerber files into a printable dimension. The board was fabricated using the combination of laser CNC Figure 5(a) and wet-etching Figure 5(b). Laser CNC uses open-source software for its layout instructions and commands to control laser intensity level upon material used. The final layout and the assembled PCB are shown below in Figure 5(c) below. 


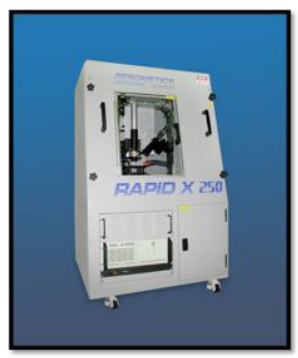

(a)

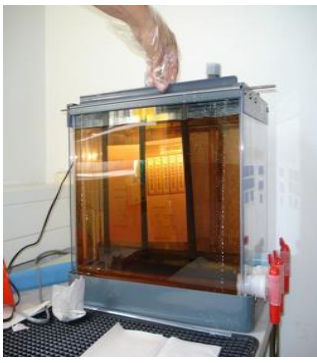

(b)

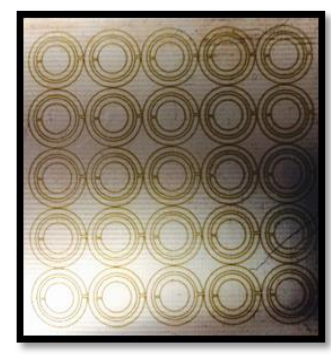

(c)

Fig. 5. (a) Resonetics Rapid X 250 laser CNC micromachining machine which allows laser cutting for any material with high precision and accuracy, (b) The outer PCB area was done with wet etching technique, and (c) the final output

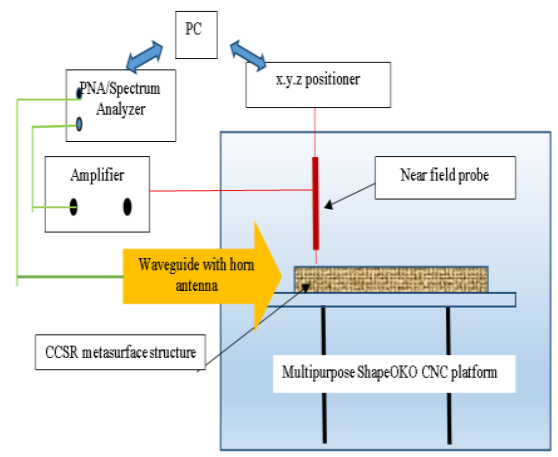

(a)

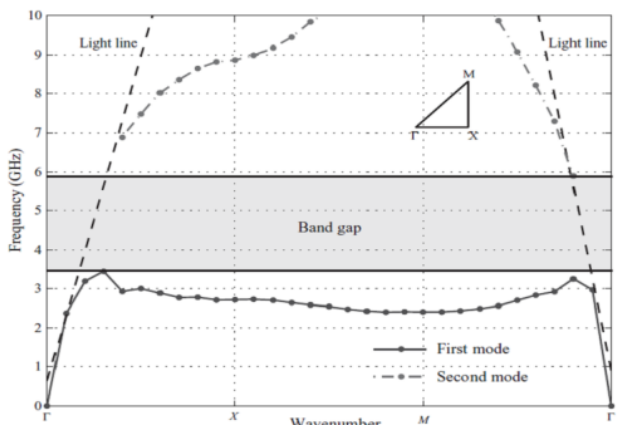

(b)

Fig. 6. Diagram of the experimental setup, (a) the LANGER EMV-Tehcnik ${ }^{\mathrm{TM}}$ near field probe, and (b) Shows the expected dispersion diagram of the CSAR structure. The vertical axis shows the frequency and the horizontal axis represents the values of the transverse wavenumbers $\left(\beta_{\mathrm{x}}, \beta_{\mathrm{y}}\right)$. Three specific points are: $\Gamma, \mathrm{X}$ and $\mathrm{M}$

Agilent E5071C network analyzer was used to measure the phase shift (angle of $\mathrm{S}_{21}$ ), insertion loss (magnitude of $S_{21}$ ), signal delay and return loss (magnitude of $S_{11}$ ). The PNA can be replaced with a much simpler equipment such as the Spectrum Analyzer since it will be easier to map E-field and H-field and plot it back using Excel ${ }^{\mathrm{TM}}$. Figure 6(a) shows the diagram of the intended platform for measuring purposes. The dispersion of a metasurfaces are tested and compared in terms of measurement, simulation and the theoretical model. The distance of the waveguide is set to $\mathrm{d}=10 \mathrm{~mm}$ from the CCSR metasurface.

\section{Expected dispersion results}

The expected results for each dispersion method are shown in Figure 6(b). The measured dispersion [3] will closely match with measured, simulation and theory. Only the lowest mode can be measured, and the results stop at $10 \mathrm{GHz}$. Above this frequency, data is difficult to obtain (due to lack of equipment and facility). The measurement probe is scanned $5 \mathrm{~mm}$ above the surface, and if significant wave power is located below this height, it becomes difficult to obtain an accurate reading.

When two or more modes are presented, it becomes difficult to determine the phase velocity [4] of any single mode due to interference between the modes. In simulation and 
theory, single mode operation can be strictly enforced, and the dispersion curves can be extended to higher frequencies that can be measured experimentally.

From Figure 6(b), the first two modes are shown as dashed lines for the theoretical solutions, and as dots for simulation. The first theory curves uses ideal impedance theory to obtain impedance at intermediate angles and the second theory curve uses simulation to get these values. A black dashed line is the dispersion of light in a vacuum. Guided modes must lie below the dispersion relation of the outer region, and this is shown as a black line. Simulated and theoretical curves are shown for the first two guided modes. The second mode has a cutoff when it intersects the dispersion curve of the outer region. The outer region dispersion represents the highest phase velocity that can be achieved in the waveguide, as this outer region has a lower index than the interior region.

\section{Expected near-field measurement}

Two-dimensional near field measurement testbed were built using the setup described in previous section. The final dimension of the CCSR surface produced was $75 \times 75 \mathrm{~mm}$. The measured sample is smaller than the total table board size to allow space for the feed and absorbers around the edge of the surface. The excitation source, shown before in Figure 6(a), does not couple perfectly into the guided modes at all frequencies, and some power is excited directly to the outer region. A vertical probe was swept $5 \mathrm{~mm}$ above the surface along a $2 \mathrm{~mm}$ grid. The probe was made to scan higher above the surface for twodimensional field measurements because the surface was not completely flat and scanning lower could cause contact between the probe and surface at the edges.

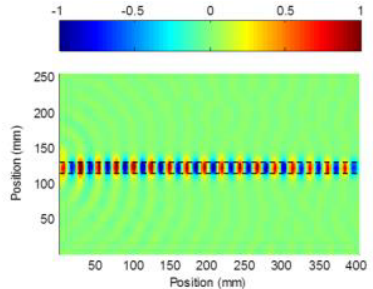

(a)

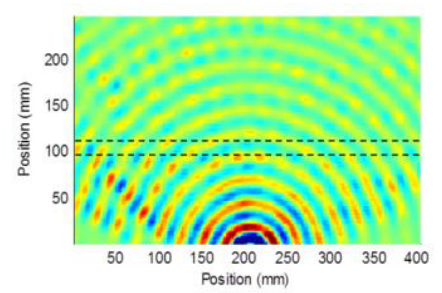

(b)

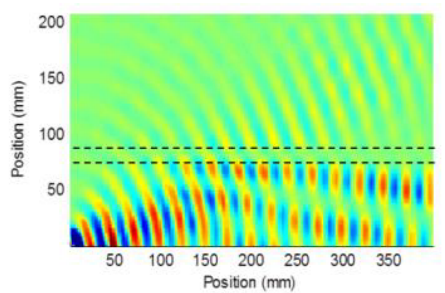

(c)

Fig. 7.Expected measured fields. The plot show normalized vertical electrical field at $5.8 \mathrm{GHz}$ for (a) guided mode, (b) orthogonal mode, and (c) angled mode. Fields in (a), (b), and (c) are not plotted to the same scale

A guided mode is displayed in Figure 7(a). The mode is bound near the guide and does not leak to the outer region. The measured bandwidth for guided mode is from 5 to $8 \mathrm{GHz}$. below $5 \mathrm{GHz}$ the measured mode is regular surface wave. At $5 \mathrm{GHz}$, as shown in Figure 7(a) almost all of the fields are in the waveguide mode and minimal fields are present in the outer region. Above $8 \mathrm{GHz}$, the source excites more measurable field in the outer region than as guided mode. This is because higher frequency modes have high impedance[5].

Figure 7(b) shows a two-dimensional field plot for a surface with a feed located in the exterior region. The feed was a vertically-oriented horn antenna that excited electromagnetic normal phase fronts on the surface. The guiding and outer regions have identical surface impedance for surface wave incident normal to the guide. Therefore, as seen in Figure 7(b), waves at normal incidence pass through the guide as if it was not present, and transparency is confirmed. In this case, the wave front is only orthogonal directly above the source. At off-normal angles, some reflections and distortion can be seen off the guide. In Figure 7(c) a trapezoidal feed is used to launch a surface wave toward the 
waveguide at a small grazing angle. In this case, the higher impedance guiding region affects a significant portion of the incident power.

The authors are grateful to the financial support by Project No 9003-00449 from Fundamental Research Grant Scheme (FRGS) 2014 from Ministry of Education (MOE), Malaysia.

\section{References}

1. J.Q. Liu, M.D. He, S. Chen, C.P. Huang, L. Zhou, Y.Y. Zhu, J. Phys.: Condens. Matter., 23, 215303 (2011)

2. O. Abu Safia, L. Talbi, K. Hettak, Magnetics, IEEE Transactions., 49, 968 (2013)

3. Y. Hao, R. Mittra, FDTD modeling of metamaterials: theory and applications. Artech house (2008)

4. H.X. Xu, G.M. Wang, M.Q. Qi, Z.M. Xu, Appl. Phys. Lett., 101, 173501 (2012)

5. D. Seetharamdoo, R. Sauleau, K. Mahdjoubi, A.C. Tarot, J. Appl. Phys., 98, 063505 (2005) 